\title{
Predicting indoor particle dispersion under dynamic ventilation modes with high-order Markov chain model
}

\author{
Xiong Mei' $(\bowtie)$, Chenni Zeng', Guangcai Gong ${ }^{2}$ \\ 1. School of Energy and Power Engineering, Changsha University of Science and Technology, 960 Wanjiali South Road, Changsha 410114, China \\ 2. School of Civil Engineering, Hunan University, 2 Lushan South Road, Changsha 410082, China
}

\begin{abstract}
Mechanical and natural ventilations are effective measures to remove indoor airborne contaminants, thereby creating improved indoor air quality (IAQ). Among various simulation techniques, Markov chain model is a relatively new and efficient method in predicting indoor airborne pollutants. The existing Markov chain model (for indoor airborne pollutants) is basically assumed as first-order, which however is difficult to deal with airborne particles with non-negligible inertial. In this study, a novel weight-factor-based high-order (second-order and third-order) Markov chain model is developed to simulate particle dispersion and deposition indoors under fixed and dynamic ventilation modes. Flow fields under various ventilation modes are solved by computational fluid dynamics (CFD) tools in advance, and then the basic first-order Markov chain model is implemented and validated by both simulation results and experimental data from literature. Furthermore, different groups of weight factors are tested to estimate appropriate weight factors for both second-order and third-order Markov chain models. Finally, the calculation process is properly designed and controlled, so that the proposed high-order (second-order) Markov chain model can be used to perform particle-phase simulation under consecutively changed ventilation modes. Results indicate that the proposed second-order model does well in predicting particle dispersion and deposition under fixed ventilation mode as well as consecutively changed ventilation modes. Compared with traditional first-order Markov chain model, the proposed high-order model performs with more reasonable accuracy but without significant computing cost increment. The most suitable weight factors of the simulation case in this study are found to be $\left(\lambda_{1}=0.7, \lambda_{2}=0.3, \lambda_{3}=0\right)$ for second-order Markov chain model, and $\left(\lambda_{1}=0.8, \lambda_{2}=0.1, \lambda_{3}=0.1\right)$ for third-order Markov chain model in terms of reducing errors in particle deposition and escape prediction. With the improvements of the efficiency of state transfer matrix construction and flow field data acquisition/processing, the proposed high-order Markov chain model is expected to provide an alternative choice for fast prediction of indoor airborne particulate (as well as gaseous) pollutants under transient flows.
\end{abstract}

\section{Keywords}

high-order Markov chain; dynamic ventilation modes; indoor particles; particle dispersion; fast prediction

\section{Article History}

Received: 12 July 2021

Revised: 28 September 2021

Accepted: 18 October 2021

(C) Tsinghua University Press and Springer-Verlag GmbH Germany, part of Springer Nature 2021

\section{Introduction}

Particulate matter has comprehensively been recognized as one of the major threats to human health (Kan et al. 2007; Zhang et al. 2021). Particles emitted from contaminant sources such as vehicles and biomass-burning may be inhaled by both adults and children (Buonanno et al. 2013; DíazRobles et al. 2014; Deng et al. 2018), and further deposition can be easily observed in human respiratory systems (Lin et al. 1994; Oravisjärvi et al. 2011; Sonnenberg et al. 2020). Since nowadays people spend near $90 \%$ of their lifetime indoors (Klepeis et al. 2001), the personal exposure level of particulate pollutants is directly determined by the quality of air within buildings. In particular, particles generated by indoor sources including tobacco smoke and cooking activities can make a substantial contribution to adverse effects on occupants (Abt et al. 2000; Wang et al. 2020). Numerous investigations have been conducted by researchers

E-mail:xmei@csust.edu.cn 
worldwide to figure out methods of reducing indoor particle concentrations (Zhao and You 2021). For instance, by enhancing aggregation/coagulation among suspended particles, large particles have a higher chance of depositing onto indoor surfaces (Guelcher et al. 2000; Rim et al. 2012) or being removed from surfaces in advance to avoid further resuspension (Mei et al. 2016). Meanwhile, particles with smaller aerodynamic diameters are also easier to be removed out of the indoor environment directly by natural or mechanical ventilation (Li and Chen 2003; Zhang and Chen 2006).

Ventilation systems in various buildings are designed to produce better indoor air quality (IAQ) by removing gaseous/granular pollutants as well as providing fresh outdoor air (Yang et al. 2021). Ventilation system types (HowardReed et al. 2003; Satheesan et al. 2020), ventilation rates (Nomura et al. 1997; Dai and Zhao 2020), and inlet/outlet locations (Zhao et al. 2004; Liu et al. 2019b; Zhou et al. 2021) of rooms are major parameters influencing ventilation system performance and IAQ. Particularly, effects of ventilation configurations on particle deposition and distribution have been extensively investigated. For instance, Zhang and Chen (2006) experimentally and numerically investigate particle transport and deposition in underfloor air distribution (UFAD) system. Compared with ceiling supply and side wall supply systems, UFAD system has been proved to have the best particle removal efficiency and the greatest potential to reduce cross-contamination. Gao and Niu (2007) have developed a three-dimensional drift-flux model for particle movements in turbulent indoor airflows and applied it to three typical ventilation systems: mixing ventilation (MV), displacement ventilation (DV), and UFAD. Rim and Novoselac (2010) conclude that the relationship between ventilation effectiveness and particle concentration varies predominantly with airflow pattern as well as particle properties in the typical indoor environment. In addition, very few investigations regarding the effects of dynamic ventilation modes on indoor particle concentrations have been reported. For example, the impact of changed ventilation rates from a fixed value to demand controlled value on IAQ in offices over six US cities has been numerically studied (Rackes and Waring 2013). In order to improve IAQ as well as reduce energy use in buildings, multi-objective optimizations have also been applied to discover suitable dynamic ventilation control strategies (Rackes and Waring 2014).

A great quantity of models have been developed to study indoor particle dynamics under different ventilation conditions. Nazaroff (2004) has summarized some modelling techniques used to study indoor particle dynamics, among which the computational fluid dynamics (CFD) is a powerful tool for predicting indoor particle distributions and deposition processes. The CFD-based models can be mathematically classified into two types, namely Eulerian and Lagrangian methods, and have been extensively adopted to perform the particle phase simulation. Each method earns its own popularity depending on the specific research objective. The Eulerian (macro) model treats particulate matter as continuum and is generally used to predict particle spatial concentration distributions (Shimada et al. 1996; Holmberg and Chen 2003; Kim et al. 2020). But the Lagrangian (micro) method is adopted to acquire evolutionary/time-resolved process of particle motion track and distribution (Lu et al. 1996; Zhang and Chen 2004; Zhao et al. 2004; Lu and Zhang 2019; Ray et al. 2020). Both Eulerian and Lagrangian methods require large numbers of computing resources, which is not acceptable for specific emergency situations such as unexpected fire hazard. To make a compromise, the so-called Markov chain model is introduced (Nicas 2000). Compared to Eulerian and Lagrangian methods, Markov chain model has been proved to be able to provide a much higher computing speed (Chen et al. 2015). Other investigations have further accelerated the calculation process (Mei et al. 2017; Liu et al. 2019a) and enabled the capacity of both predicting gravity-induced particle deposition (Mei and Gong 2018) and estimating air pollution source strength (Li et al. 2020). In the above-mentioned Markov chain-related investigations, there is one important assumption: Markov chain model is a first-order model. This assumption emphasizes that any future state is only determined by the current state, which is not always valid under specific conditions. For large size particles under transient flow conditions (or dynamic ventilation modes), the inertial force (as well as deposition behavior) of particles cannot be neglected, and particles may not follow the airflow constantly as flow field changes rapidly. Thus some of the future states may be determined by both the current state and a limited number of its previous state(s), which means high-order Markov chain model is needed. To the authors' best knowledge, however, little research on dispersion of indoor particles under dynamic ventilation modes by high-order Markov chain model has ever been reported. In addition, the effects of different orders of Markov chain model on model performance remain unknown.

The present study aims to investigate particle distribution and deposition within a limited number of space(s) under dynamic ventilation modes by a newly developed high-order Markov chain model. The high-order Markov chain model is creatively proposed to address the problem when any future particle spatial distribution is not only determined by the present particle spatial distribution. The general procedure of the present study is to solve flow field in advance by using CFD tools (In this study, Fluent is adopted), and then the proposed Markov chain models are implemented/realized 
with self-developed codes in MATLAB platform to perform particle-phase simulation. More specifically, a first-order Markov chain model is firstly presented and further validated by simulated results as well as experimental data from literature. Then a high-order Markov chain model is developed based on previous first-order Markov chain model, and several groups of weight factors for second-order and thirdorder models are tested to find the most suitable combinations. Finally, the proposed second-order Markov chain model (with tested most suitable weight factors) is implemented to simulate particle dispersion and deposition under dynamic ventilation modes. Gravitational force is considered as the dominant factor causing particle deposition in this study.

\section{Methods}

2.1 Markov chain model and its modification for transient airflow

The so-called Markov chain model is a probabilistic model, which can be used to predict indoor particle contaminant dispersion and distribution, first introduced by Nicas (2000). Assuming that a target space is divided into $n$ subspaces (cells), in which temporal distributions of particle contaminants can be obtained as follows: the Markov chain model connects the present state $S_{t}$, which represents how many particles are in those cells at time $t$, with any future state $\boldsymbol{S}_{t+\Delta t}$ at time $t+\Delta t$ by a so-called state transfer matrix $\boldsymbol{P}$. The above relationship can be described as:

$\boldsymbol{S}_{t+\Delta t}=\boldsymbol{S}_{t} \boldsymbol{P}$

where $\boldsymbol{S}_{t}$ and $\boldsymbol{S}_{t+\Delta t}$ are state vectors of the same length $n . \boldsymbol{P}$ is an $n \times n$ probability matrix. The state transfer matrix $\boldsymbol{P}$ contains two types of probabilities, namely the probability of a particle remaining in the current cell $i\left(p_{i i}\right)$ and the probability of a particle moving from cell $i$ to a neighboring cell $j\left(p_{i j}\right)$ after time $\Delta t$. Apparently, the state transfer matrix $\boldsymbol{P}$ can be constructed by arranging each probability $p_{i j}$ and $p_{i i}$ to corresponding positions in Eq. (2) (Chen et al. 2014). Probabilities $p_{i j}$ and $p_{i i}$ can be calculated by Eqs. (3) and (4), respectively (Nicas 2000).

$$
\begin{aligned}
& \boldsymbol{P}=\left[\begin{array}{cccc}
p_{11} & p_{12} & \cdots & p_{1 n} \\
p_{21} & p_{22} & \cdots & p_{2 n} \\
\cdots & \cdots & \cdots & \cdots \\
p_{n 1} & p_{n 2} & \cdots & p_{n n}
\end{array}\right] \\
& p_{i i}=\exp \left(-\sum_{n b} \frac{Q_{i(n b)}}{V_{i}} \Delta t\right) \\
& p_{i j}=\frac{Q_{i j}}{\sum_{n b} Q_{i(n b)}}\left(1-p_{i i}\right)
\end{aligned}
$$

where $Q_{i(n b)}$ denotes the fluid flow rate from cell $i$ to its all neighboring cells, and $Q_{i j}$ represents the fluid flow rate from cell $i$ to cell $j$. All flow rates are composed of two parts: the mean flow rate $Q_{i j}$ mean and the turbulent fluctuating flow rate $Q_{i j \_f l u c t u a t i n g}$, in which the particle turbulence diffusion is considered indirectly. Equation (2) gives the expression of state transfer matrix $\boldsymbol{P}$ that could be used in an enclosed environment. But for the ventilated environment, escaped particles should be stored separately in order to fulfill the requirement of mass conservation theory. Therefore, an extra cell numbered as $n+1$ is attached to the computational domain to receive particles escaped from any cells that have direct connections with outlet(s). The original state transfer matrix $\boldsymbol{P}$ then can be extended and expressed as:

$$
\boldsymbol{P}=\left[\begin{array}{ccccc}
p_{11} & p_{12} & \cdots & p_{1 n} & p_{1(n+1)} \\
p_{21} & p_{22} & \cdots & p_{2 n} & \cdots \\
\cdots & \cdots & \cdots & \cdots & \cdots \\
p_{n 1} & \cdots & \cdots & p_{n n} & \cdots \\
\cdots & \cdots & \cdots & \cdots & p_{(n+1)(n+1)}
\end{array}\right]
$$

where $p_{i(n+1)}$ is the probability of a particle getting out of the computational domain from cell $i$. Based on the mass conservation theory, all the probabilities in matrix $\boldsymbol{P}$ should be no less than zero, and the sum of all the elements in each row is equal to 1 . Escaped particles are not allowed to move out of cell $n+1$, which means $p_{(n+1)(n+1)}$ is equal to 1 . It also should be mentioned that Eq. (1) represents a single-step state transfer process within one time step $\Delta t$. As for any desired multi-step transfer process under steady-state flow field, which corresponds to a fixed state transfer matrix $\boldsymbol{P}$, the calculation problem then can be addressed by the following equation:

$S_{t+k \Delta t}=S_{t} P^{k}$

where the index $k$ denotes the steps that a specific transfer process needs to reach the target state. Detailed information about the multi-step transfer can be found in the research conducted by Mei et al. (2017). In a ventilated room with transient flow field (or under consecutively changed ventilation modes), the state transfer matrix $\boldsymbol{P}$ cannot be considered as constant. But within a sufficiently small time period of a transient flow, the flow field can accordingly be assumed as steady-state. If the simulated transient flow process is divided into the limited number of steady-state sub-periods, $m$ ones for instance, thus the state transfer process of airborne particles (or any other gaseous contaminants) can be calculated by the following equation:

$\boldsymbol{S}_{t+k \Delta t}=\boldsymbol{S}_{t} \boldsymbol{P}_{1}^{k_{1}} \boldsymbol{P}_{2}^{k_{2}} \cdots \boldsymbol{P}_{m}^{k_{m}}$

where $\boldsymbol{P}_{1}-\boldsymbol{P}_{m}$ are state transfer matrices corresponding to 
the sub-periods mentioned above, which are defined based on the steady-state flow fields within each sub-periods. Positive numbers $k_{1}-k_{m}$ are the quantities of time steps $(\Delta t)$ required for state transfer within each sub-period. The sum of $k_{1}-k_{m}$ is equal to $k$. Since particles with specific diameters $(\geq 5 \mu \mathrm{m})$ will be used in this study for simulation, gravitational forceinduced particle deposition should be considered. Compared with traditional concentration (Eulerian model) or trajectory (Lagrangian model) based models (Wang et al. 2012), the basic Markov chain model in Eq. (7), however, can only solve the fluid flow induced particle transmission. To address this problem, two key steps should be followed: (1) Adjust the original computational domain to receive and store deposited particles. Some deposited particles receiving cells are placed adjacent to boundary cells of the original computational domain, once suspended particles are transferred to boundary cells, the additionally added cells will play the role of solid surface to receive and "store" deposited particles. (2) Adjust the original state transfer matrix $\boldsymbol{P}$ to include the effect of gravity by implementing (within each time period) the algorithm proposed by Mei and Gong (2018). The probability adjustment makes sure that particles can vertically fall down through cells (including the transfer process from boundary cells to those additionally added cells). To avoid redundancy, detailed information is not repeated here, readers who are interested may refer to the previous publication (Mei and Gong 2018).

\subsection{High-order Markov chain model}

As elaborated above, the contaminant distributions in the computational domain at any time $t$ can be regarded as a single (distribution) state, and the transport of airborne contaminants then describes the state-changing process from one to another. Based on the work by Ching et al. (2003), the so-called high-order Markov chain model for a specific computational domain with $k$ cells can be described as follows:

$\boldsymbol{S}_{t+n+1}=\sum_{i=1}^{n} \lambda_{i} \boldsymbol{P}_{i} \boldsymbol{S}_{t+n+1-i}$

$\sum_{i=1}^{n} \lambda_{i}=1, \lambda_{i} \geq 0$

where $S_{t+n+1}$ is a $k \times 1$ vector denoting the state at time $t+n+1 . \boldsymbol{P}_{i}$ is the state transfer matrix described above $(k \times k)$ with its column sum being equal to 1 , and $\lambda_{i}$ is the weight factor used to balance past and present states. To give a clear example as well as avoid potential high computational cost at the very beginning, the order $n$ is temporarily assumed to be 2 , and Eq. (8) can be simplified as:

$\boldsymbol{S}_{t+n+1}=\lambda_{1} \boldsymbol{P}_{1} \boldsymbol{S}_{t+n}+\lambda_{2} \boldsymbol{P}_{2} \boldsymbol{S}_{t+n-1}$
For a steady-state flow field, $\boldsymbol{P}$ is a fixed matrix which means $\boldsymbol{P}_{1}=\boldsymbol{P}_{2}=\boldsymbol{P}$. Transpose both sides of Eq. (10) we have:

$$
\boldsymbol{S}_{t+n+1}{ }^{\mathrm{T}}=\lambda_{1} \boldsymbol{S}_{t+n}{ }^{\mathrm{T}} \boldsymbol{P}_{1}^{\mathrm{T}}+\lambda_{2} \boldsymbol{S}_{t+n-1}{ }^{\mathrm{T}} \boldsymbol{P}_{2}^{\mathrm{T}}=\lambda_{1} \boldsymbol{S}_{t+n}{ }^{\mathrm{T}} \boldsymbol{P}^{\mathrm{T}}+\lambda_{2} \boldsymbol{S}_{t+n-1}{ }^{\mathrm{T}} \boldsymbol{P}^{\mathrm{T}}
$$

To establish contact with previous contents, Eq. (11) can be further rewritten as:

$\boldsymbol{S}_{t+\Delta t}=\lambda_{1} \boldsymbol{S}_{t} \boldsymbol{P}+\lambda_{2} \boldsymbol{S}_{t-\Delta t} \boldsymbol{P}$

where $S_{t+\Delta t}$ and $S_{t}$ all are $1 \times k$ vectors representing particle distribution state for the whole computational domain at time $t+\Delta t$ and $t$, respectively. For the third-order Markov chain model, the corresponding state transfer process can be deduced as follows:

$\boldsymbol{S}_{t+\Delta t}=\lambda_{1} \boldsymbol{S}_{t} \boldsymbol{P}+\lambda_{2} \boldsymbol{S}_{t-\Delta t} \boldsymbol{P}+\lambda_{3} \boldsymbol{S}_{t-2 \Delta t} \boldsymbol{P}$

For transient airflow, which can be divided into a limited number of short time periods with steady-state flow fields, the so-called second-order Markov chain model can be applied within or between each short time period. The state transfer process between and within each short time periods can accordingly be expressed as Eqs. (14) and (15), respectively.

$$
\begin{aligned}
& \boldsymbol{S}_{t_{1}+\Delta t}=\lambda_{1} \boldsymbol{S}_{t_{1}} \boldsymbol{P}_{2}+\lambda_{2} \boldsymbol{S}_{t_{1}-\Delta t} \boldsymbol{P}_{1} \\
& \boldsymbol{S}_{t_{1}+2 \Delta t}=\lambda_{1} \boldsymbol{S}_{t_{1}+\Delta t} \boldsymbol{P}_{2}+\lambda_{2} \boldsymbol{S}_{t_{1}} \boldsymbol{P}_{2}
\end{aligned}
$$

where $\boldsymbol{P}_{1}$ and $\boldsymbol{P}_{2}$ are state transfer matrices of two consecutive short time periods, and time $t_{1}$ is the duration of the first one of two consecutive short time periods. The primary goal of the present study is to investigate the possibility of using high-order Markov chain under transient flow, which is simplified here by three consecutively changed ventilation modes to predict particle dispersion and deposition. It is easy to analyze that, under transient flow, Markov chain model with higher $(\geq 3)$ order is needed only if the time step size $\Delta t$ (typically should be larger than particle relaxation time) defined in Eq. (1) exceeds any short time periods with steadystate flow fields. To theoretically estimate appropriate weight factors under steady-state flow (let us take the second-order Markov chain model for example), we have tried to solve the following revised optimization problem (Chvatal 1983)

$$
\begin{aligned}
& \hat{\boldsymbol{X}}=[\underbrace{\frac{1}{k}, \frac{1}{k}, \ldots, \frac{1}{k}, \frac{1}{k}}_{k}] \\
& \min _{\lambda} \omega, \omega \geq 0, \sum_{i=1}^{2} \lambda_{i}=1, \lambda_{i} \geq 0
\end{aligned}
$$

$\left[\begin{array}{l}\omega \\ \omega\end{array}\right] \geq \hat{\boldsymbol{X}}-[\boldsymbol{Q} \hat{\boldsymbol{X}} \mid \boldsymbol{Q} \hat{\boldsymbol{X}}]\left[\begin{array}{l}\lambda_{1} \\ \lambda_{2}\end{array}\right]$ 


$$
\left[\begin{array}{l}
\omega \\
\omega
\end{array}\right] \geq-\hat{\boldsymbol{X}}+[\boldsymbol{Q} \hat{\boldsymbol{X}} \mid \boldsymbol{Q} \hat{\boldsymbol{X}}]\left[\begin{array}{l}
\lambda_{1} \\
\lambda_{2}
\end{array}\right]
$$

where $\hat{\boldsymbol{X}}$ is the proportion of the occurrence of each contaminant distribution state (considered to be unique), which can be expressed as Eq. (16) for a pulsed (or dynamic) contaminant source. For a constant contaminant source, Eq. (16) still can be implemented until the contaminant concentration reaches constant (by then no more prediction is needed). $\boldsymbol{Q}$ is the state transfer matrix under steady-state flow, which is fixed and unique. After solving the equation sets, however, we found that " $\lambda_{1}+\lambda_{2}=1, \lambda_{1}, \lambda_{2} \geq 0$ " is the only condition required to obtain the minimum $\omega$. Therefore, it is necessary to test different combinations of $\lambda_{1}$ and $\lambda_{2}$ to obtain the most suitable weight factors (approximate range) for the present case. And several groups of combination values of $\lambda_{1}, \lambda_{2}$, and $\lambda_{3}$ for both second-order and thirdorder Markov chain models are listed in Table 1 and Table 2, respectively. The condition described in Eq. (9) for $\lambda_{1}, \lambda_{2}$ and $\lambda_{3}$ should also be satisfied.

Table 1 Tested weight factors $\lambda_{1}, \lambda_{2}$, and $\lambda_{3}$ for the second-order Markov chain model

\begin{tabular}{cccc}
\hline & $\lambda_{1}$ & $\lambda_{2}$ & $\lambda_{3}$ \\
\hline Case A1 & 1 & 0 & 0 \\
Case A2 & 0.9 & 0.1 & 0 \\
Case A3 & 0.8 & 0.2 & 0 \\
Case A4 & 0.7 & 0.3 & 0 \\
Case A5 & 0.6 & 0.4 & 0 \\
Case A6 & 0.5 & 0.5 & 0 \\
Case A7 & 0.4 & 0.6 & 0 \\
Case A8 & 0.3 & 0.7 & 0 \\
Case A9 & 0.2 & 0.8 & 0 \\
Case A10 & 0.1 & 0.9 & 0 \\
\hline
\end{tabular}

Table 2 Tested weight factors $\lambda_{1}, \lambda_{2}$, and $\lambda_{3}$ for the third-order Markov chain model

\begin{tabular}{cccc}
\hline & $\lambda_{1}$ & $\lambda_{2}$ & $\lambda_{3}$ \\
\hline Case B1 & $0.9-0.1\left(\Delta^{*}=0.1\right)$ & $0.05-0.45(\Delta=0.05)$ & $0.05-0.45(\Delta=0.05)$ \\
Case B2 & $0.05-0.45(\Delta=0.05)$ & $0.9-0.1(\Delta=0.1)$ & $0.05-0.45(\Delta=0.05)$ \\
Case B3 & $0.05-0.45(\Delta=0.05)$ & $0.05-0.45(\Delta=0.05)$ & $0.9-0.1(\Delta=0.1)$ \\
Case B4 & 0.9 & $0.01-0.09(\Delta=0.01)$ & $0.09-0.01(\Delta=0.01)$ \\
Case B5 & 0.5 & $0.1-0.4(\Delta=0.1)$ & $0.4-0.1(\Delta=0.1)$ \\
Case B6 & 0.1 & $0.1-0.8(\Delta=0.1)$ & $0.8-0.1(\Delta=0.1)$ \\
Case B7 & $0.01-0.09(\Delta=0.01)$ & 0.9 & $0.09-0.01(\Delta=0.01)$ \\
Case B8 & $0.1-0.4(\Delta=0.1)$ & 0.5 & $0.4-0.1(\Delta=0.1)$ \\
Case B9 & $0.1-0.8(\Delta=0.1)$ & 0.1 & $0.8-0.1(\Delta=0.1)$ \\
Case B10 & $0.01-0.09(\Delta=0.01)$ & $0.09-0.01(\Delta=0.01)$ & 0.9 \\
Case B11 & $0.1-0.4(\Delta=0.1)$ & $0.4-0.1(\Delta=0.1)$ & 0.5 \\
Case B12 & $0.1-0.8(\Delta=0.1)$ & $0.8-0.1(\Delta=0.1)$ & 0.1
\end{tabular}

${ }^{*} \Delta$ denotes a fixed interval size between each groups of $\lambda_{1}, \lambda_{2}$, and $\lambda_{3}$, for instance in Case B1, groups of $\left(\lambda_{1}=0.9, \lambda_{2}=0.05, \lambda_{3}=0.05\right)$ and $\left(\lambda_{1}=0.8\right.$, $\left.\lambda_{2}=0.1, \lambda_{3}=0.1\right)$

\subsection{Validation case and numerical procedure}

In order to validate the proposed models, a ventilated cubic chamber is used in the present study. Figure 1 shows the configuration of the simulated chamber with sides $L$. The chamber has four openings on both the $x+$ surface and $x-$ surface, which are of the same size $h \times h$ and symmetric about the central panel $(y=L / 2)$. The so-called "Top-Bottom" or "TB" ventilation mode denotes airflow entering from Opening 1 and exhausting through Opening 4. Another two ventilation modes "Top-Top" ("TT") and "Bottom-Top" ("BT") are also defined in a similar way. Detailed information about the basic configuration of the ventilated chamber based on the investigation by Bouilly et al. (2005) is given in Table 3.

Particles are uniformly distributed in the chamber at the initial stage. Two size groups of spherical solid particles, namely 5 and $10 \mu \mathrm{m}$, are utilized during the particle phase simulations. The density of particles is set to be $1000 \mathrm{~kg} / \mathrm{m}^{3}$. Two types of air change rates 0.5 and $1.0 \mathrm{~h}^{-1}$ are adopted. Before the flow fields are modelled by CFD tools, the chamber is divided into $45 \times 47 \times 48$ control volumes to meet the requirement of grid independency. Since the flow field simulation is not our primary concern, detailed modelling procedures are not presented. Readers who are interested could refer to a similar modelling case in research conducted by Mei et al. (2017). Once the steady-state flow field is established, the flow field/grid information data will be exported out as independent data files. Some self-developed codes as well as flow field data/grid information files then will be imported into the MATLAB environment to realize particle phase simulation. As mentioned above, gravityinduced particle deposition is considered. To evaluate the

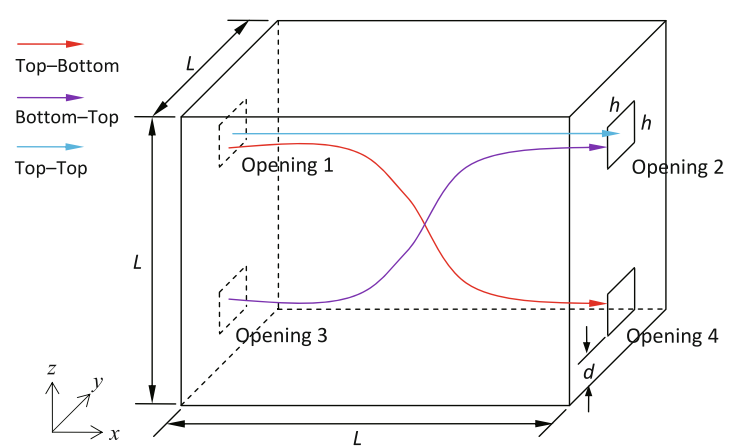

Fig. 1 Configuration of the ventilated chamber and the locations of all inlets and outlets

Table 3 Real dimensions of the ventilated chamber

\begin{tabular}{ccccc}
\hline Ventilation mode & Inlet-outlet location & $d(\mathrm{~m})$ & $h(\mathrm{~m})$ & $L(\mathrm{~m})$ \\
\hline "Bottom-Top" or "BT" & Openings 3-2 & 0.265 & 0.07 & 2.5 \\
"Top-Bottom" or "TB" & Openings 1-4 & 0.265 & 0.07 & 2.5 \\
"Top-Top" or "TT" & Openings 1-2 & 0.265 & 0.07 & 2.5 \\
\hline
\end{tabular}


intensity of the deposition process, the so-called particle deposition velocity is adopted in this study, which can be expressed as the following equation (Bouilly et al. 2005):

$V_{\mathrm{de}}=\frac{\lambda_{\mathrm{de}}}{3600} \times \frac{V}{S}$

where $V$ is the chamber volume, and $S$ is the chamber (inner) surface area. $\lambda_{\mathrm{de}}$ represents the particle deposition loss rate, which can be calculated by the following equation:

$\lambda_{\mathrm{de}}=\lambda_{\mathrm{g}}-\lambda_{\mathrm{v}}$

where $\lambda_{\mathrm{v}}$ is the air change rate, and $\lambda_{\mathrm{g}}$ is the overall loss rate which will be determined by the regression of the decreased parts of the overall particle concentration curves (Bouilly et al. 2005).

To realize the particle phase simulation with the highorder Markov chain model under dynamic ventilation modes, the ventilation mode is assumed to change in the following order: "Top-Bottom" $\rightarrow$ "Top-Top" $\rightarrow$ "Bottom-Top". Detailed flow chart of the modelling process for particle phase is presented in Figure 2. State $\boldsymbol{S}_{t 0}$ is the initial particle distribution state (vector) for particle phase calculation, states $S_{t 1}, S_{t 2}, S_{t 3}$ denote the particle distribution states (vectors) at 400,800 , and $1200 \mathrm{~s}$, respectively. It is worth noting that the change of ventilation mode (and the flow field) is assumed to be instant. Both the overall particle concentration and deposited particles are monitored during the simulation.

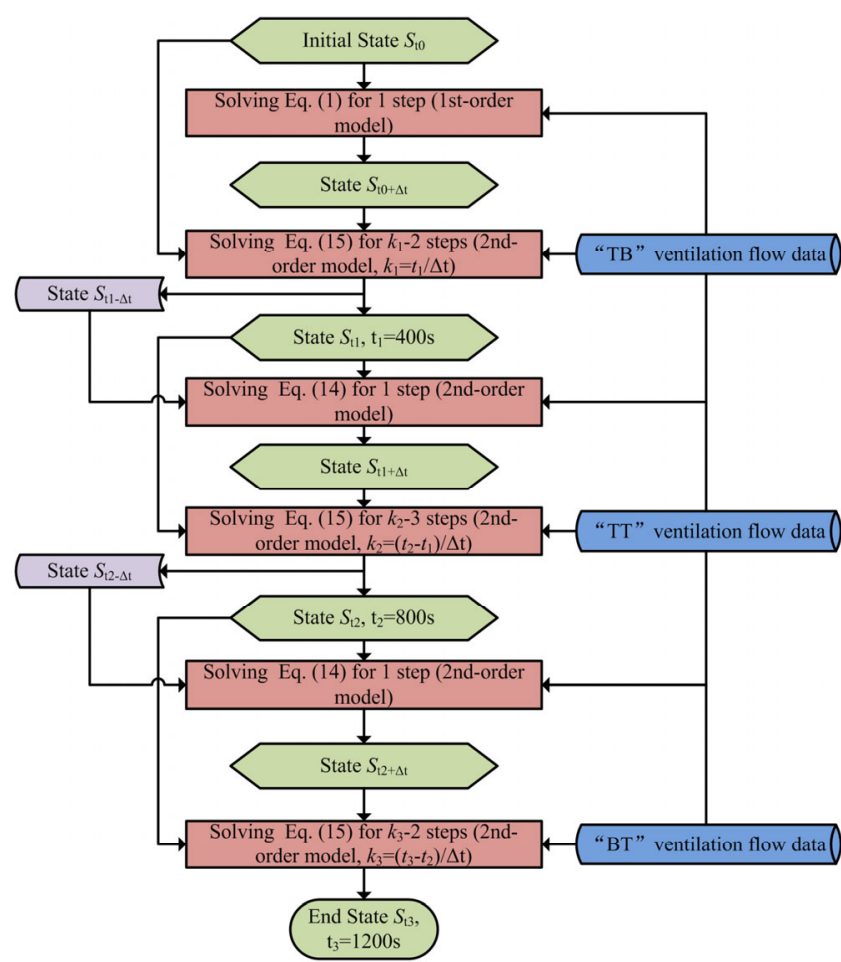

Fig. 2 Flow chart of particle-phase modelling process under dynamic ventilation mode
In the present study, dispersion and deposition of $10 \mu \mathrm{m}$ particles under the overall dynamic ventilation mode is simulated for $1200 \mathrm{~s}$ (real time), and each ventilation mode is simulated for $400 \mathrm{~s}$ (real time). But for a single ventilation mode, $600 \mathrm{~s}$ (real time) is adopted for particle phase simulation.

\section{Results}

\subsection{Basic first-order Markov chain model validation}

The basic first-order Markov chain model is used to simulate particle distributions under different ventilation modes and particle sizes, and the normalized particle concentrations are presented in Figure 3. As can be seen in Figure 3, normalized particle concentration generally decreases over time and the discrepancies induced by particle size are more significant than that by the air change rate. Comparing all decay curves of $10 \mu \mathrm{m}$ particles from Figures 3(a) to 3(c), decreasing rate for $\mathrm{ACH}=0.5 \mathrm{~h}^{-1}$ is much larger than that for $\mathrm{ACH}=1 \mathrm{~h}^{-1}$ in "Top-Bottom" ventilation mode. However, the tendency becomes inversed in both "Bottom-Top" and "Top-Top" ventilation modes. It is also evident to observe the discrepancies between different air change rates in the "Top-Bottom" ventilation mode, which is believed to be more sensitive to air change rates for particles with the diameter of $5 \mu \mathrm{m}$. Besides, the decreasing rates of all concentration decay curves for $5 \mu \mathrm{m}$ particles are almost constant while the decreasing rates for $10 \mu \mathrm{m}$ particles generally decrease over time.

As mentioned above, the deposition loss rate is calculated by Eq. (21) and the overall loss rates for three different ventilation modes ("TB", "BT", and "TT") are obtained by the regression of the decay curves presented in Figure 3. Comparisons between simulated deposition loss rates and data from literature (Bouilly et al. 2005) are listed in Table 4. As can be seen, deposition loss rates of $5 \mu \mathrm{m}$ particles are slightly underestimated by the proposed first-order model. Evident discrepancies can be found for $\mathrm{ACH}=1.0$, and the largest difference lies in the "Top-Top" ventilation mode. A reasonable explanation could be: as particle diameter $D_{\mathrm{p}}$ increases, particle deposition velocity $V_{\text {de }}$ dramatically increases to a critical value that makes the airflow velocity negligible. Then the gravitational force induced deposition turns dominant, and the realistic deposition velocity approaches to Eq. (20) in a so-called "quasi-static" air. The deposition loss rate can therefore be well predicted by the proposed first-order model under a lower air change rate for larger particles.

To have a comprehensive understanding of particle dispersion and deposition process with first-order Markov chain model, the accumulated concentration, deposition, and escape curves of $10 \mu \mathrm{m}$ particles are validated against data 

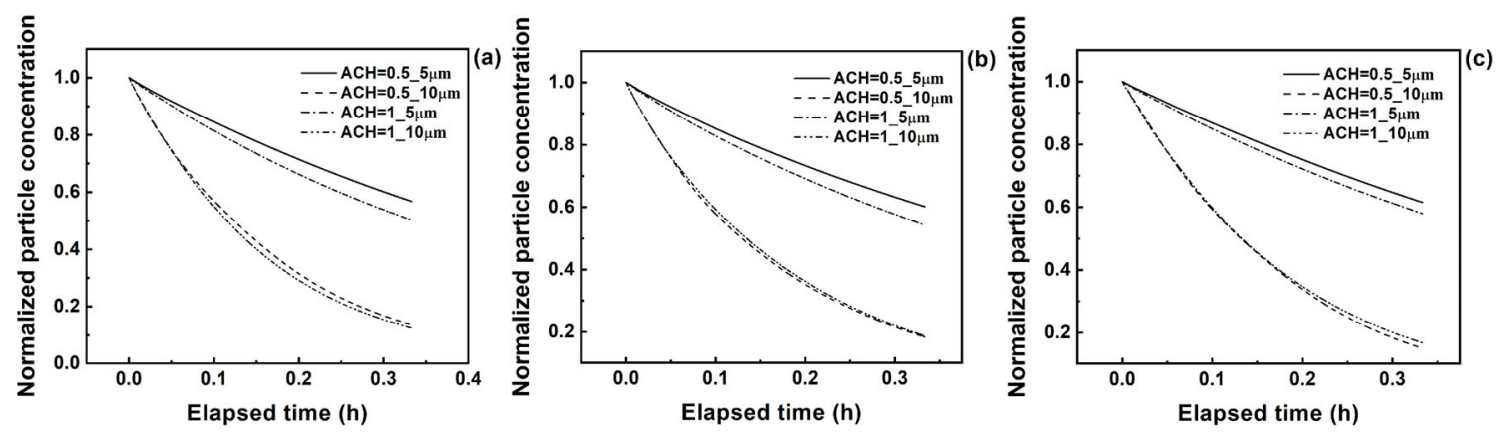

Fig. 3 Normalized particle concentration vs. time in the ventilated chamber under (a) "Top-Bottom", (b) "Bottom-Top", and (c) “Top-Top" ventilation modes

Table 4 Validation of first-order Markov chain model predicted deposition loss rate against literature data (Bouilly et al. 2005)

\begin{tabular}{|c|c|c|c|c|c|c|}
\hline \multirow[b]{2}{*}{ Deposition loss rate $\left(\mathrm{h}^{-1}\right)$} & \multicolumn{2}{|c|}{ "Bottom-Top" } & \multicolumn{2}{|c|}{ “Top-Bottom" } & \multicolumn{2}{|c|}{ “Тop-Тop” } \\
\hline & $\mathrm{ACH}=0.5$ & $\mathrm{ACH}=1.0$ & $\mathrm{ACH}=0.5$ & $\mathrm{ACH}=1.0$ & $\mathrm{ACH}=0.5$ & $\mathrm{ACH}=1.0$ \\
\hline $5 \mu \mathrm{m}$, literature simulation data & 1.30 & 1.13 & 1.62 & 2.23 & 1.11 & 1.38 \\
\hline $10 \mu \mathrm{m}$, literature simulation data & 4.68 & 4.24 & 4.65 & 5.49 & 4.81 & 4.47 \\
\hline $4-5 \mu \mathrm{m}$, literature experiment data & $1.11-1.38$ & $1.24-1.63$ & $0.99-2.01$ & $1.42-1.85$ & $0.85-1.40$ & $0.86-1.36$ \\
\hline 7.5-10 $\mu \mathrm{m}$, literature experiment data & $3.24-3.99$ & $2.1-4.26$ & $2.39-5.03$ & $2.78-4.88$ & $2.15-4.00$ & $2.47-5.50$ \\
\hline $5 \mu \mathrm{m}$, present model & 1.05 & 0.85 & 1.19 & 1.06 & 0.94 & 0.63 \\
\hline $10 \mu \mathrm{m}$, present model & 4.76 & 4.12 & 5.27 & 5.12 & 4.90 & 4.26 \\
\hline
\end{tabular}

from literature as shown in Figure 4. Meanwhile, predicted deposition velocity is also validated against experimental data from literature, which is illustrated in Figure 5. As depicted in Figure 4, the results of overall concentration and escaped particle fractions agree well with the simulated results provided by Bouilly et al. (2005). But the deposited particle fraction is gradually overestimated over time for "Top-Bottom" ventilation mode.

For two particle size groups of 5 and $10 \mu \mathrm{m}$ in Figure 5, predicted deposition velocities are within the scope of the experiment results provided by Lai (2002) and Bouilly et al. (2005). Moreover, obvious consistence can be found between

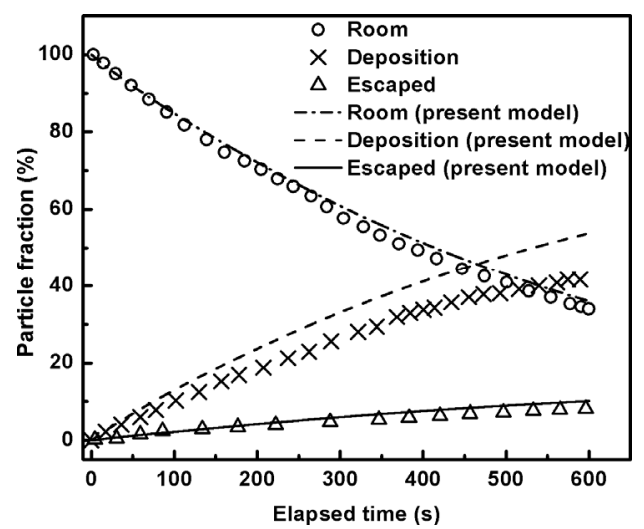

Fig. 4 Comparison of normalized $10 \mu \mathrm{m}$ particle fraction of chamber concentration, deposition, and escape against the data from literature (Bouilly et al. 2005) under “Top-Bottom" ventilation mode when $\mathrm{ACH}=1.0$ the predicted results and data from literature (Bouilly et al. 2005) for $5 \mu \mathrm{m}$ particles. It is also demonstrated in Figure 5 that agreements between some of the ventilation modes are good (such as "Bottom-Top" ventilation mode) whereas some show significant discrepancies ("Top-Bottom" ventilation mode and "Top-Top" ventilation under ACH = 1.0). It is also worth noting that symbols in the left part of Figure 5, which are not in accord with symbols presented in the right part of Figure 5, are used to distinguish the data of our proposed model and the data from corresponding literature.

\subsection{Second- and third- order Markov chain models under steady-state airflow}

Predicted evolutionary fractions of suspended, deposited, and escaped $10 \mu \mathrm{m}$ particles in the studied chamber by second-order Markov chain model are demonstrated in Figure 6. The "TB" ventilation mode is adopted for both second-order and third-order Markov chain models. Totally ten cases (from Case A1 to Case A10) are included in Figure 6. As can be seen in Figure 6, the simulated suspended particle fraction in Case A1 (first-order Markov chain model) agrees well with the data from literature. Simulation results of deposited and escaped particle fractions in Case A4 (secondorder Markov chain model, $\lambda_{1}=0.7, \lambda_{2}=0.3, \lambda_{3}=0$ ), however, are much closer to the validation data. But the suspended particle fraction is overestimated up to approximately $10 \%$ (until $600 \mathrm{~s}$ ) compared with the predictions in Case A1, 

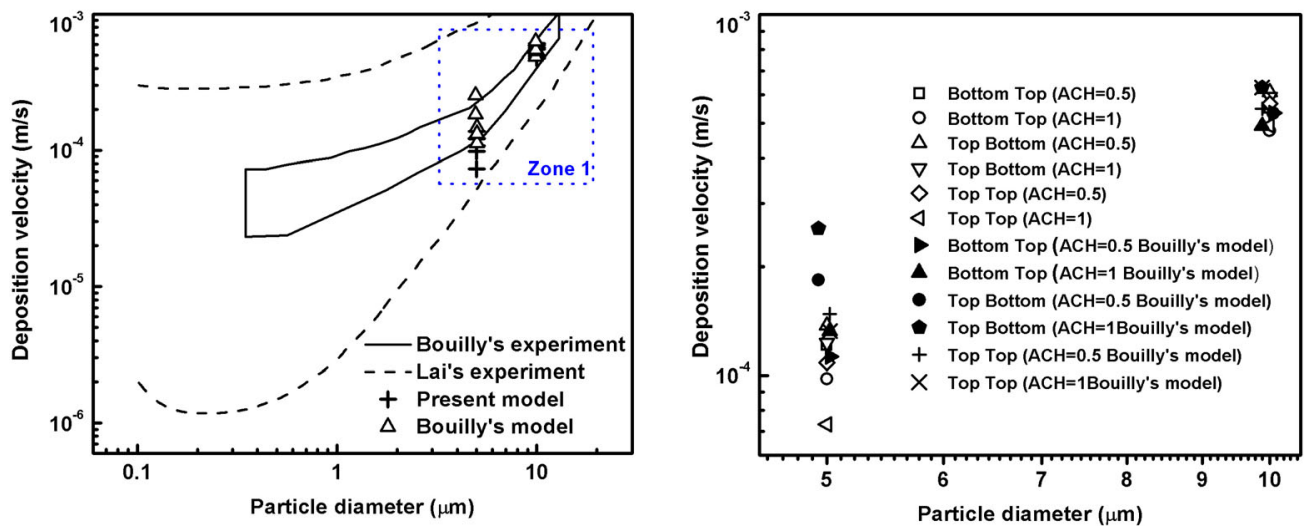

Fig. 5 Comparison of the predicted deposition velocity with experimental results from literature (Lai 2002; Bouilly et al. 2005), left part shows the overall comparison, and the right part shows the enlarged Zone 1 in the left ("Top-Bottom", 10 $\mu \mathrm{m}, \mathrm{ACH}=1.0$ )
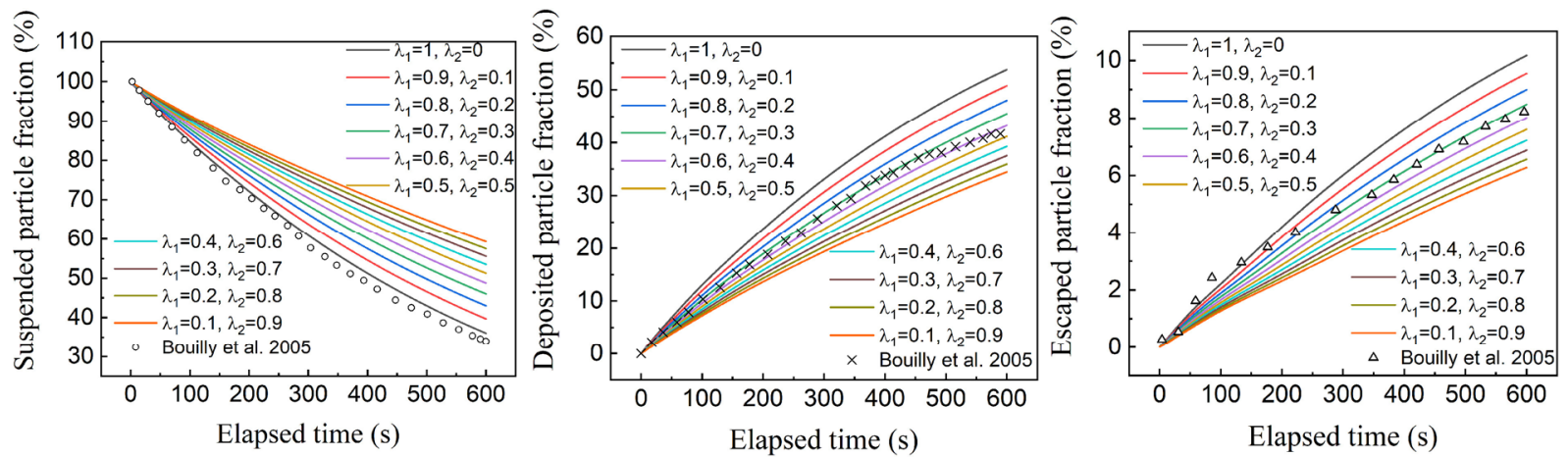

Fig. 6 Predicted suspended (left), deposited (middle), and escaped (right) particle fractions vs. time by second-order Markov chain model (Cases A1-A10)

which is obviously acceptable for particulate matters. If we choose Case A2 (second-order Markov chain model, $\lambda_{1}=$ $0.9, \lambda_{2}=0.1, \lambda_{3}=0$ ) and Case A3 (second-order Markov chain model, $\lambda_{1}=0.8, \lambda_{2}=0.2, \lambda_{3}=0$ ), the maximum discrepancies between simulated results and validation data would be controlled within 5\%. In particular, as shown in escaped particle fraction curves, none of these ten cases can capture the trends precisely before around $300 \mathrm{~s}$.

Predicted evolutionary fractions of suspended, deposited, and escaped $10 \mu \mathrm{m}$ particles in the studied chamber by thirdorder Markov chain model are demonstrated in Figures 7-10. More specifically, Figure 7 contains the results of Cases B1-B3, in which weight factor $\lambda_{1}$ changes from 0.9 to 0.1 , and the rest two weight factors $\lambda_{2}$ and $\lambda_{3}$ are equal to each other. As depicted in the deposited and escaped curves of Case B1 in Figure 7, the combination of weight factors $\lambda_{1}=$ $0.8, \lambda_{2}=0.1, \lambda_{3}=0.1$ gives a relatively good agreement between simulated results and validation data. But there is still up to around $10 \%$ error for suspended particle fraction results. Variations of $\lambda_{2}$ and $\lambda_{3}$ from 0.9 to 0.1 in Cases B2 and B3 fail to show any consistency with validation data from literature (Bouilly et al. 2005). Deposited and suspended particles account for the majority of total particles after $600 \mathrm{~s}$, thus discrepancies between simulated results and validation data of escaped particles are generally acceptable in any simulation cases of the present study.

In Case groups B4-B6, B7-B9, and B10-B12, $\lambda_{1}, \lambda_{2}$, and $\lambda_{3}$ are set to change between $0.9,0.5$, and 0.1 , respectively. The rest weight factors in those groups are set based on the same regulation as described in Table 2. So these three case groups are tested to evaluate the influence of two dynamic weight factors and one fixed weight factor. Cases B1-B3 are designed to test the effects of three dynamic weight factors. With one fixed weight factor (for instance, $\lambda_{3}$ ) being equal to zero, which has been displayed in Table 1 and tested previously in Figure 6, the third-order Markov chain model then turns into second-order Markov chain model.

When $\lambda_{1}$ decreases from 0.9 to 0.1 , as depicted from left to right in Figure 8, the overall discrepancies of simulation results between third-order and first-order Markov chain models increases. As $\lambda_{1}$ decreases from 0.9 to 0.1 in Figures 9 and 10, however, the tendency reverses (the average discrepancy decreases but the largest discrepancy almost remains unchanged). The range (sector area) of cluster of curves is basically determined by the fixed interval non-dimensional size $\Delta$ defined in Table 2 , and the range decreases with the 

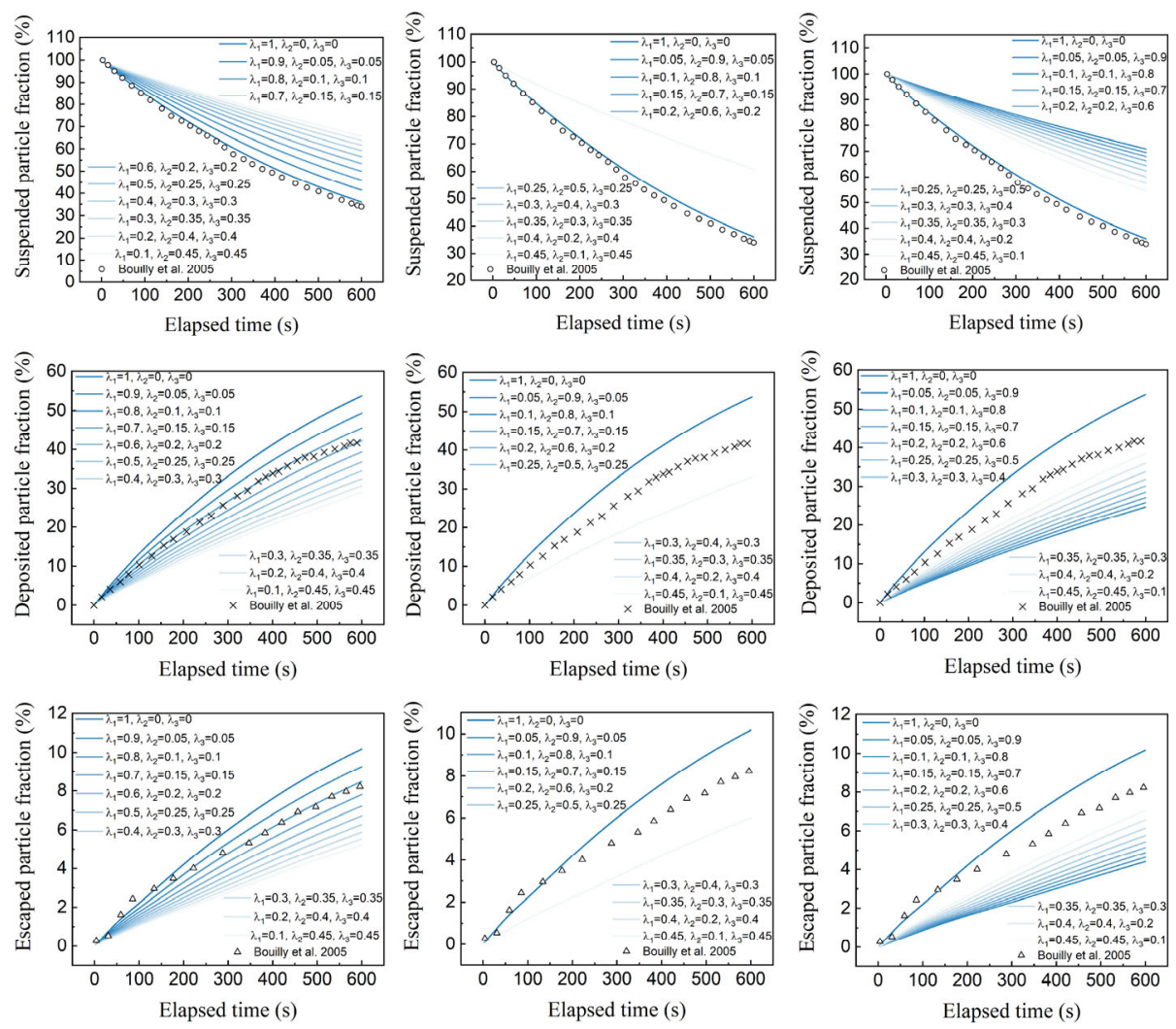

Fig. 7 Predicted suspended (up), deposited (middle), and escaped (down) particle fractions vs. time by third-order Markov chain model for Case B1 (left), Case B2(middle), and Case B3 (right)
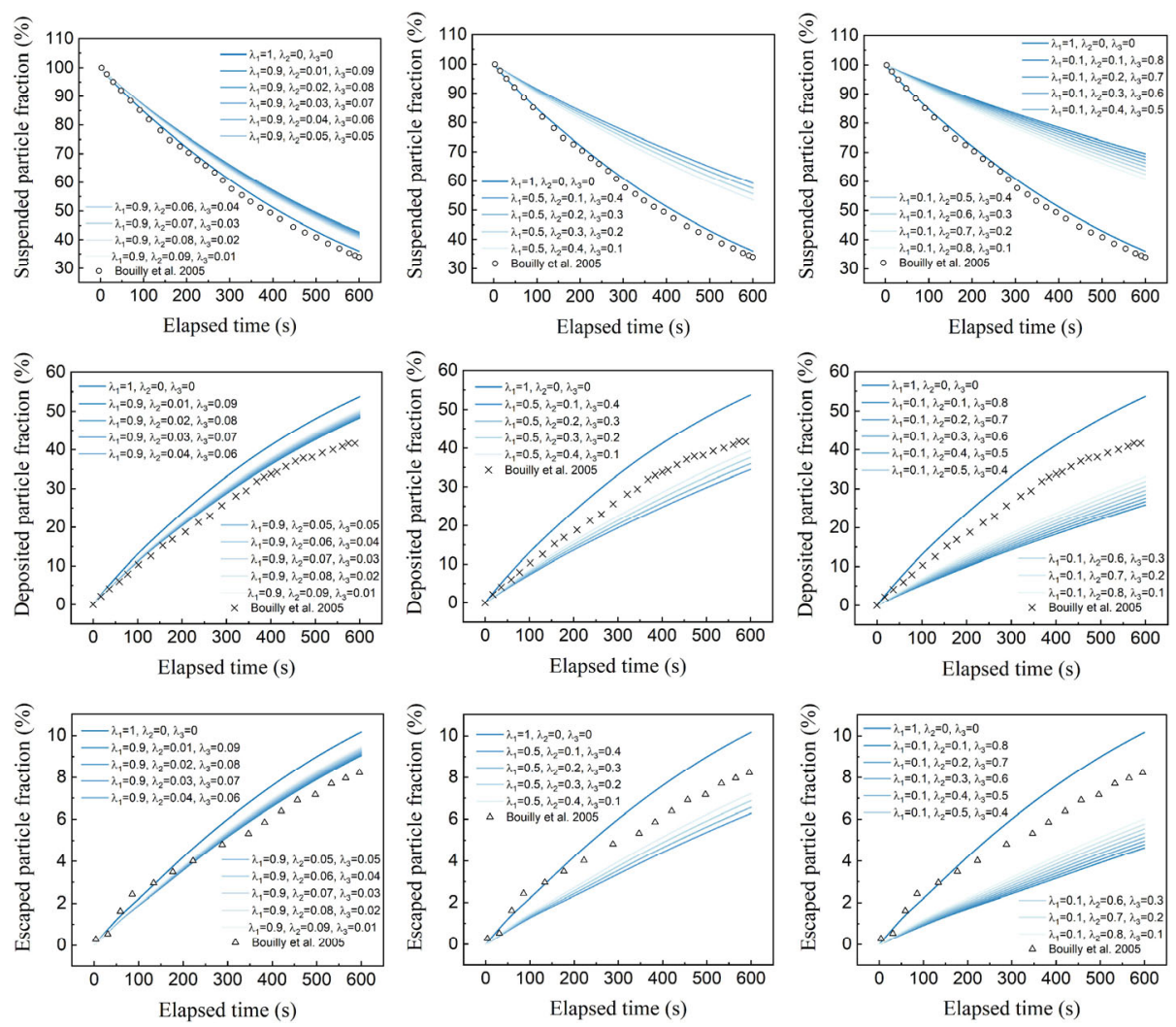

Fig. 8 Predicted suspended (up), deposited (middle), and escaped (down) particle fractions vs. time by third-order Markov chain model for Case B4 (left), Case B5(middle), Case B6 (right) 

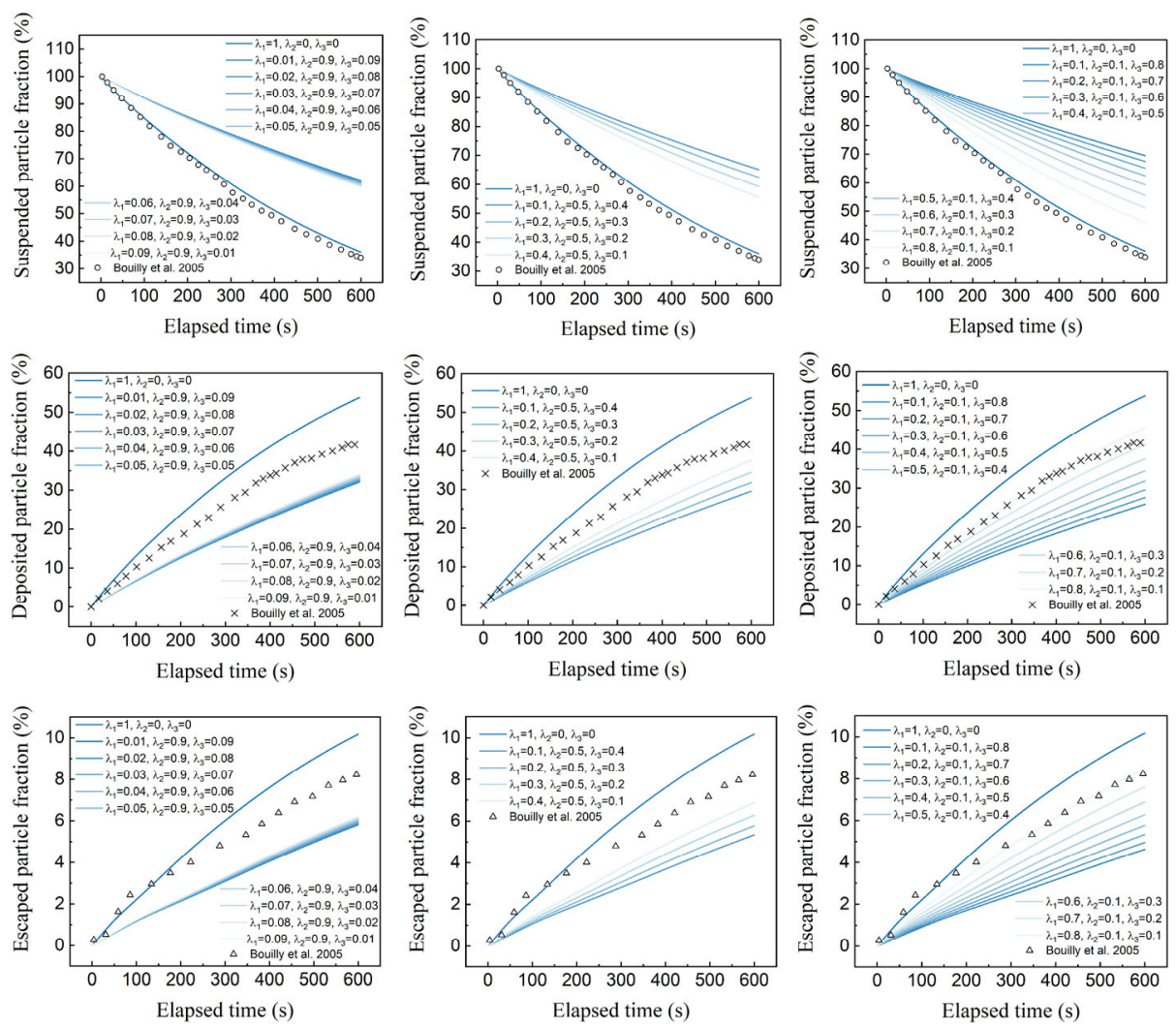

Fig. 9 Predicted suspended (up), deposited (middle), and escaped (down) particle fractions vs. time by third-order Markov chain model for Case B7 (left), Case B8(middle), and Case B9 (right)
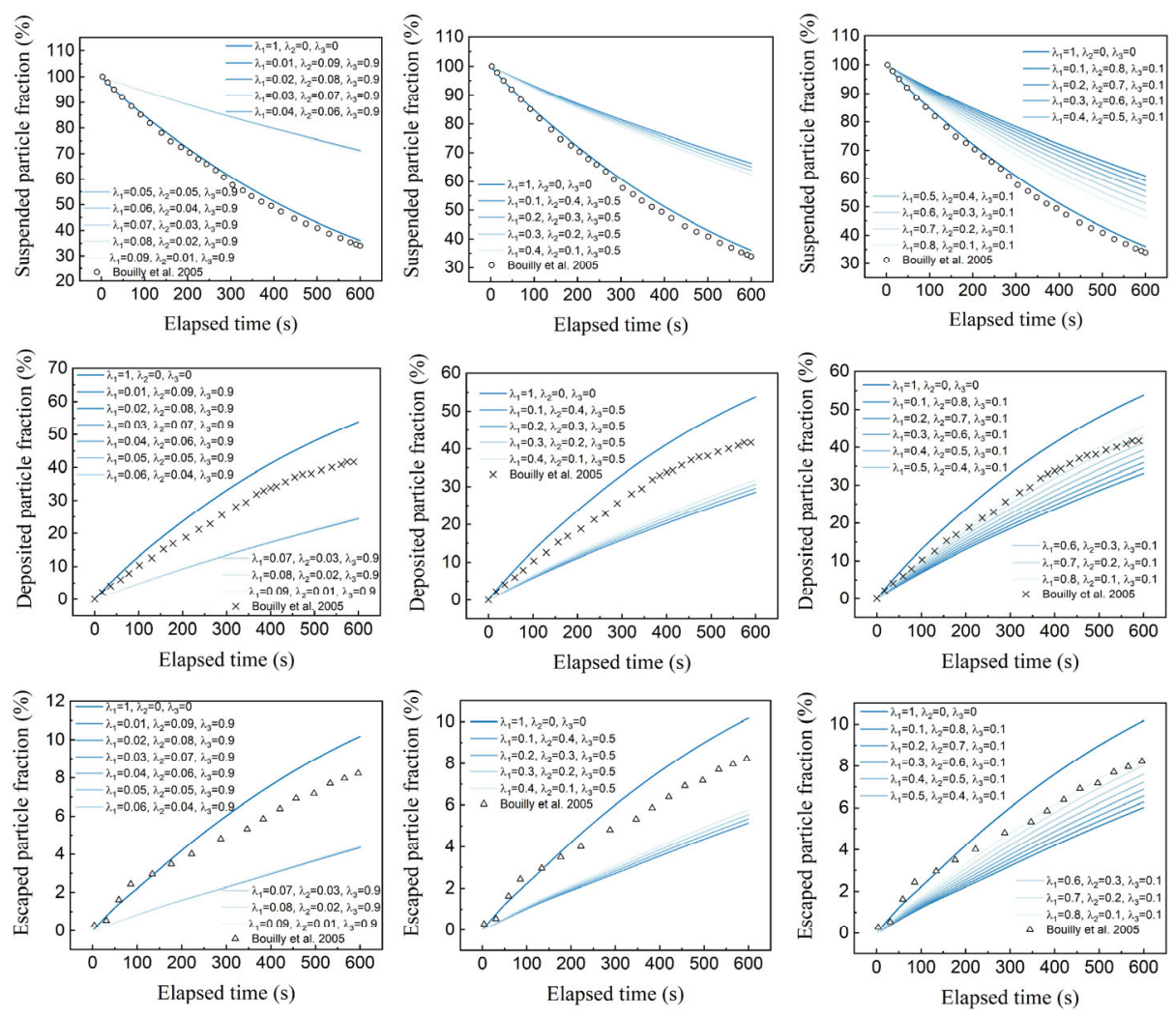

Fig. 10 Predicted suspended (up), deposited (middle), and escaped (down) particle fractions vs. time by third-order Markov chain model for Case B10 (left), Case B11(middle), and Case B12 (right) 
decrease of $\Delta$. Good agreements of deposited and escaped particle fractions can be found in Case B9 $\left(\lambda_{1}=0.8, \lambda_{2}=0.1\right.$, $\left.\lambda_{3}=0.1\right)$ and Case B12 $\left(\lambda_{1}=0.8, \lambda_{2}=0.1, \lambda_{3}=0.1\right)$, but there still are up to around $10 \%$ error for suspended particle fractions. If the discrepancies between validation data and simulated results of third-order model exceed the differences between validation data and simulated results of first-order model, corresponding cases (such as Cases B6, B7, and B10) are not considered as a reasonable option to find suitable weight factors. It is reasonable to deduce that the simulated results by third-order Markov chain model are more sensitive to $\lambda_{1}$ rather than $\lambda_{2}$ or $\lambda_{3}$.

\subsection{Second-order Markov chain model under dynamic ventilation modes}

Predicted evolutionary fractions of suspended, deposited, and escaped $10 \mu \mathrm{m}$ particles in the studied chamber under dynamic ventilation modes are demonstrated in Figure 11, Figure 12, and Figure 13, respectively. As explained in

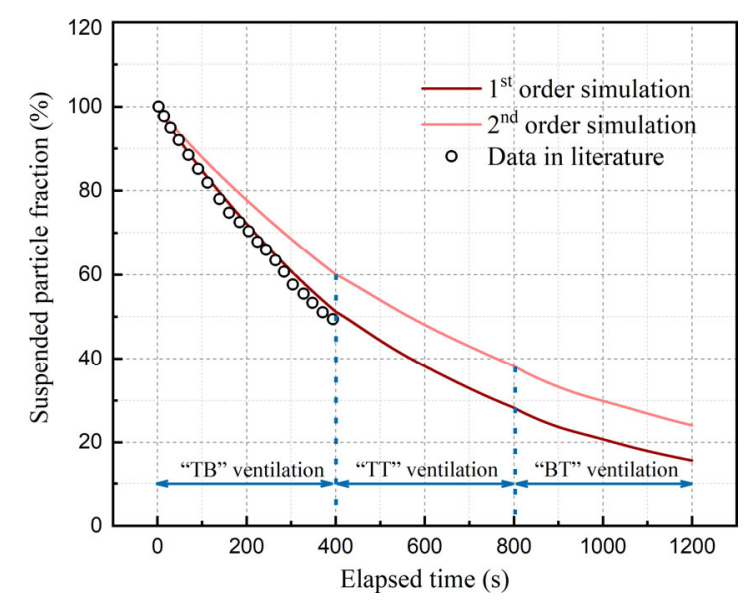

Fig. 11 Suspended $10 \mu \mathrm{m}$ particle fractions vs. time for dynamic ventilation modes

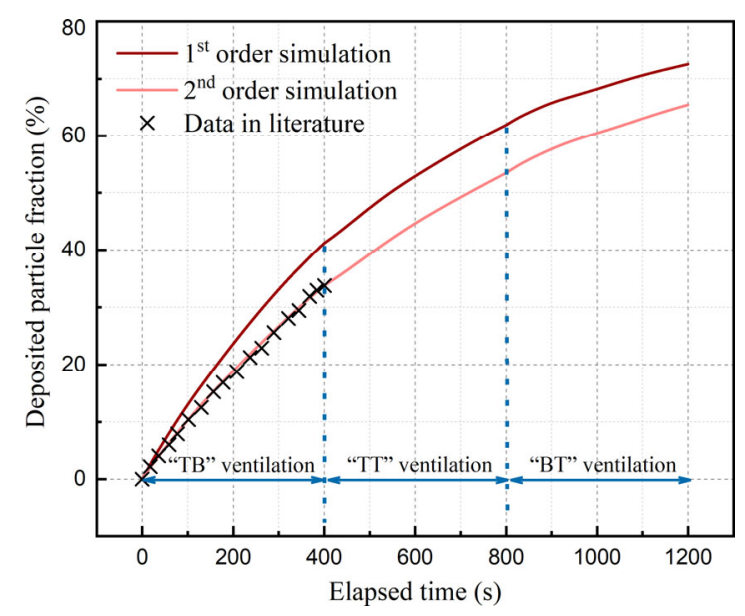

Fig. 12 Deposited $10 \mu \mathrm{m}$ particle fractions vs. time for dynamic ventilation modes

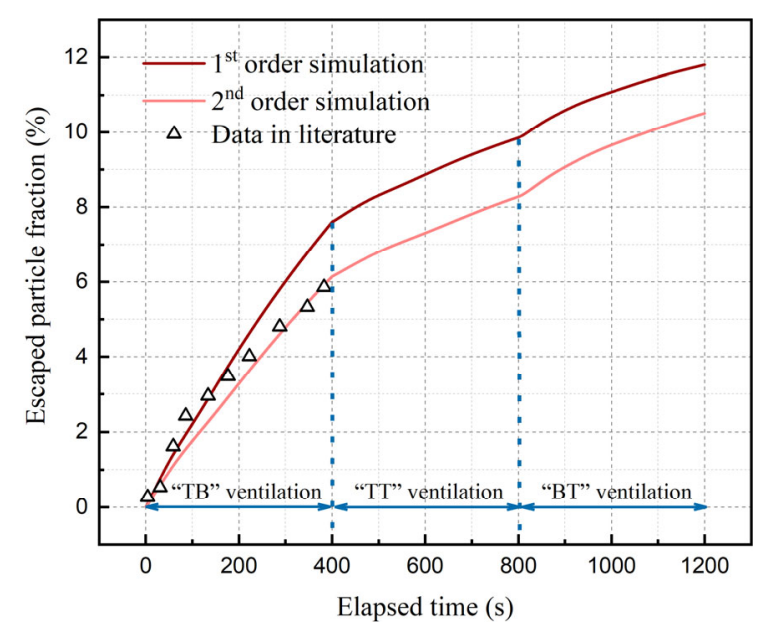

Fig. 13 Escaped $10 \mu \mathrm{m}$ particle fractions vs. time for dynamic ventilation modes

Section 2.2, the second-order rather than a third-order Markov chain model is used. Based on our previous results in Figure 6 of Section 3.2, corresponding suitable weight factors of the second-order Markov chain model are set as $\lambda_{1}=0.7, \lambda_{2}=0.3$. It is evident that different ventilation mode combinations would result in different particle dispersions. As mentioned in the method section, the adopted ventilation mode combination can be divided into three sections, i.e., “Top-Bottom" ventilation mode, “Top-Top" ventilation mode, and "Bottom-Top" ventilation mode. If the duration of each ventilation mode can be gradually shortened, from another perspective, the flow condition under dynamic ventilation modes could be treated as transient.

As depicted in Figure 11, the largest concentration decrease rate can be found under the "Top-Bottom" ventilation mode while the smallest decrease rate is observed under the "BT" ventilation mode. For deposition curves, the largest and smallest increase rates are observed during the "TB" and "BT" ventilation modes, respectively. As previously presented in Figure 3, the decreasing rate of $10 \mu \mathrm{m}$ particle concentration would also decrease over time with a fixed ventilation mode. It is necessary to know whether the variation in Figure 11 is caused by ventilation mode or time. To be in consistent with the results in Figure 3, the first-order Markov chain model is used to simulate continuously for $1200 \mathrm{~s}$ (real time) under “TB” and “TB-TT-BT" ventilation modes. Results are given in Figure 14.

As can be seen from Figure 14, both curves decrease over time. But the overall decreasing rate of $10 \mu \mathrm{m}$ particle concentration under specific dynamic ventilation mode is smaller than that of steady-state ventilation mode. Thus, we would say that the decay rate of suspended particles decreases over time and also can be influenced by ventilation mode.

For escaped curves, however, the largest and smallest increase rates are found under "TB" and "TT" ventilation 


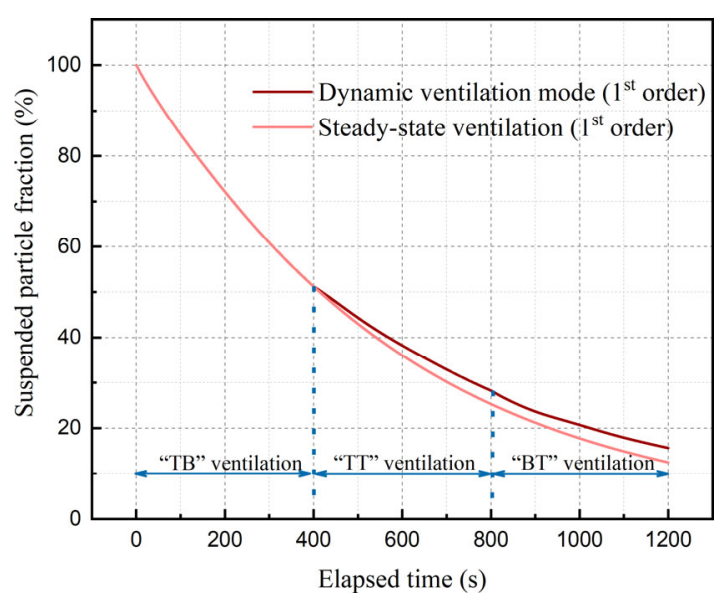

Fig. 14 Suspended $10 \mu \mathrm{m}$ particle fractions vs. time for specific dynamic ventilation mode ("TB-TT-BT") and steady-state ventilation mode (“TB”)

modes, respectively. It is evident to see that "TB" is the most efficient ventilation mode in removing particles out of the chamber (regardless of deposition or ventilation). The overall predictive results of second-order Markov chain model are generally smaller than that predicted by first-order Markov chain model for deposited and escaped particle fractions. But for suspended particle fraction, the tendency reverses. The differences between first-order and second-order predicted results would gradually increase during the "TopBottom" ventilation mode, then the differences tend to remain constant during the "TT" and "BT" ventilation modes. The discrepancies between first- and second-order Markov chain models during the "TT" and "BT" ventilation modes are around $10 \%, 8 \%$, and $1.8 \%$ for suspended, deposited, and escaped particle fractions, respectively. If particles with small relaxation time are uniformly distributed before ventilation, the combination order of ventilation modes (for instance, "TB" $\rightarrow$ "TT" $\rightarrow$ "BT" or "BT" $\rightarrow$ “TT" $\rightarrow$ “TB") would have less effect on final escaped particles with a fixed air change rate compared with particles with larger relaxation time. For the present study, however, particles are assumed to be $10 \mu \mathrm{m}$, and deposition behavior accounts for a large proportion of suspended particles reduction.

\section{Discussion}

Despite the computing efficiency of the proposed model in predicting particle deposition and dispersion under dynamic ventilation modes (The overall calculation consumes about 960 s), there still exist several limitations for the present study. For instance, the proposed first-order Markov chain model shows better capability in modelling suspended particles which has been validated in Figure 4. But deposited (along with escaped) particles are overestimated, which is in line with the assumption that the dispersion and deposition of large particles are determined not only by the present state (airflow) but also by limited number of former states (inertial force or gravity). As shown in Table 4, however, the proposed first-order model can provide a reasonable prediction on deposition loss rate only for particles with a larger diameter $(10 \mu \mathrm{m})$. That is because the particle settling velocity presented in Eq. (20) is assumed to be primarily valid in a "quasi-static" fluid environment, so the proposed first-order model may also be sensitive to airflow intensity. Besides, only two particle size groups ( 5 and $10 \mu \mathrm{m}$ ) are tested in this study, thus only a qualitative conclusion about whether the proposed model is valid for small or large particles can be drawn. And the exact particle diameter range is still uncertain, which also deserves further investigation.

One of the main purposes of this study is to apply the proposed high-order model to dynamic ventilation modes. Simulated results show that the proposed second-order Markov chain model can provide good agreements with the validation data in terms of deposition and escape. But there still exists approximately up to $10 \%$ (more precisely, $12 \%$ ) discrepancy for suspended particle fraction, which means there is still room for model accuracy improvement. If one expects relatively balanced predictions among suspended, deposited and escaped particles, i.e. the least variations of all three errors, suitable weight factors between $\left(\lambda_{1}=0.8\right.$, $\left.\lambda_{2}=0.2, \lambda_{3}=0\right)$ and $\left(\lambda_{1}=0.9, \lambda_{2}=0.1, \lambda_{3}=0\right)$ can be applied to the second-order Markov chain model. And discrepancies between simulated results and validation data will be maintained within around $7.5 \%$ (smaller than $12 \%$ for suspended particles when $\lambda_{1}=0.7, \lambda_{2}=0.3, \lambda_{3}=0$ ) for both suspended and deposited particles. As to the third-order Markov chain model, let us take Case B1 for instance, weight factors of $\left(\lambda_{1}=0.9, \lambda_{2}=0.05, \lambda_{3}=0.05\right)$ can be implemented and corresponding maximum discrepancies can be reduced to around $7.5 \%$ and $6.9 \%$ for suspended and deposited particles, respectively. Apparently, it is the current state that affects future state most since suitable values of $\lambda_{1}$ generally exceed 0.7 in the present study. It is also worth noting that, based on the state transfer process of high-order Markov chain model, the most suitable weight factors identified in the present study are case-dependent since the state transfer matrix $\boldsymbol{P}$ for various cases would change rapidly (for instance from $\boldsymbol{P}$ to $\mathbf{0}$ ) under real transient flow conditions. The highorder Markov chain model is developed to address the problem when any future particle spatial distribution is not only determined by the present particle spatial distribution, and gravity induced large particle deposition is a typical example. To make the deposition phenomenon more significant and easy to validate, both $5 \mu \mathrm{m}$ and $10 \mu \mathrm{m}$ particles are therefore adopted. Compared with large particles, movements of small particles $(<1 \mu \mathrm{m})$ are primarily dominated by airflow in the gravitational field. Deposition 
process of small particles $(<1 \mu \mathrm{m})$ is less significant indoors and usually can only be observed under specific conditions including considerable temperature gradient and electric fields. Therefore, we have tested another thermophoresis induced particle deposition case, which is previously investigated with first-order Markov chain model (Mei et al. 2019). In the present study, the basic simulation principle of thermophoresis-induced particle deposition is similar to that of the gravitational force induced particle deposition. To model particle deposition onto surfaces under the effects of thermophoresis, refer to the previous description in Section 2.1, two key steps also should be done: (1) Adjust the original computational domain to receive and store deposited particles. Some deposited particles receiving cells are placed adjacent to all boundary cells of the original computational domain, once suspended particles are transferred to boundary cells, the additionally added cells will play the role of solid surface to receive and "store" thermophoresis induced deposited particles. (2) Adjust the original state transfer matrix $\boldsymbol{P}$ to include the effect of thermophoresis by implementing (within each time period) the algorithm proposed in study conducted by Mei et al. (2019). which is based on the conversion from thermophoresis (or thermophoretic velocity) to particle deposition flow rates. The probability adjustments make sure that particles can move from high-temperature cells to low-temperature cells (including the transfer process from boundary cells to those additionally added cells). To avoid redundancy, more detailed information is not repeated here, readers who are interested may refer to the previous publication (Mei et al. 2019).

The experimental validation data originate from the research conducted by Tsai and $\mathrm{Lu}$ (1995), which focuses on a plate-to-plate thermophoretic precipitator for submicron particles. Two parallel plates of the precipitator have the same size of $70 \mathrm{~mm}$ in length and $30 \mathrm{~mm}$ in width, and the gap between these two plates is $0.38 \mathrm{~mm}$. The top and the bottom plates are heated and cooled by two constant sources, respectively. A constant temperature gradient is therefore established between the two plates. The temperature gradient is set to be 503 and $837 \mathrm{~K} / \mathrm{cm}$ for the $\mathrm{NaCl}$ particles, 526 and $879 \mathrm{~K} / \mathrm{cm}$ for sodium fluorescein particles. The investigated diameters of both types of particles are 0.04, 0.06, 0.08, 0.1, $0.2,0.3,0.4$ and $0.5 \mu \mathrm{m}$. More detailed information can be found in the original reference (Tsai and Lu 1995).

As illustrated in Figure 15, the second-order Markov chain model can improve prediction accuracy to some extent. Here the "collect efficiency" is the fraction of deposited particles due to thermophoretic force in one specific direction. Discrepancies between different combinations of parameters $\lambda_{1}$ and $\lambda_{2}$ for different particle sizes are quite small. However, the most suitable weight factors for this case are $\lambda_{1}=0.9$, $\lambda_{2}=0.1$. To avoid redundancy and excess of page limitation, detailed information is not repeated here. It is well known that indoor particle deposition process can be influenced (but not necessarily at the same time) by mechanisms such as Brownian diffusion, turbulent diffusion, gravitational settling, thermophoresis, and electrophoretic force (for charged particles). As mentioned above, gravitational force and thermophoresis induced particle deposition can be considered. The electrophoretic force, as another field force similar to gravitational force, can also be considered in a similar way if needed. Turbulent diffusion, as explained in Section 2.1, has already been taken into consideration indirectly. The Brownian diffusion, however, cannot be considered in the present model currently. This is indeed the limitation of our newly proposed model, which should be further investigated to expand its application.

In Section 3.3, all flow field data of three ventilation modes are pre-acquired from several independently calculated steady-state flow fields. The proposed model, theoretically, can be used to simulate particle dispersion under transient flow fields by repeating the procedure described in Figure 2. But the computing speed will be remarkably limited by the traditional flow field calculation, reconstruction of the state transfer matrix within each time step and the frequent data exchanges between the proposed model and CFD software.
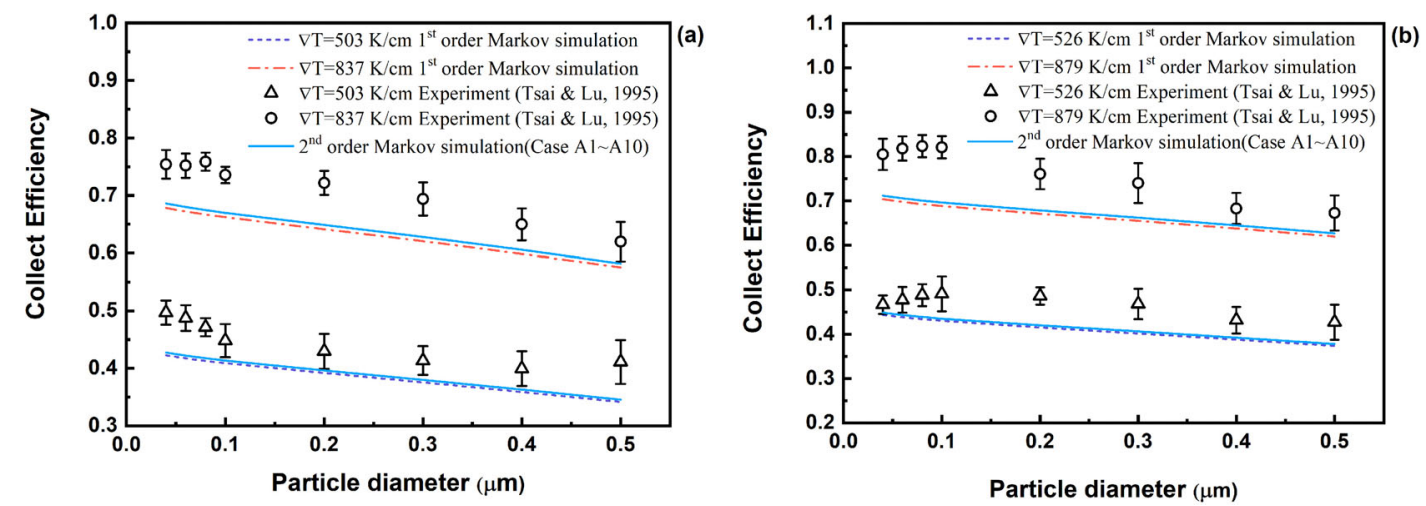

Fig. 15 Predicting thermophoresis induced (a) $\mathrm{NaCl}$ and (b) sodium fluorescein particles deposition with high-order Markov chain model 
The whole computing process mainly includes three parts: original flow field/grid data processing, state transfer matrix construction, and state transfer calculation. The overall computing speed discrepancies among different order Markov chain models are not significant (about $322 \mathrm{~s}$ simulation time for real $1200 \mathrm{~s}$ real "TB" ventilation). But in the "state transfer calculation" part, where different state transfer equations are solved, the computing time would slightly increase over order (from about 1.12 to $1.74 \mathrm{~s}$ ). It is worth noting that the computing time (322 s) is less than the real transmission time $1200 \mathrm{~s}$ and the so-called faster-than-realtime prediction has already been realized in these cases. It is also estimated that both the state transfer matrix construction and the data exchange (original flow field/grid data processing) account for more than $99 \%$ of the overall computing time (around $954 \mathrm{~s}$ simulation time for real $1200 \mathrm{~s}$ "TB-TT-BT" ventilation). Processing flow field/grid data and constructing matrix $\boldsymbol{P}$ repeatably is the main obstacle to realize comprehensive application of the proposed model under transient flow field (such as under emergency situations in large complex buildings). Thus, further efforts should be made in optimizing matrix construction process as well as the data exchange process. For instance, (faster-than) realtime flow field simulation techniques including but not limited to fast fluid dynamics (FFD), online-offline flow field database, and limited critical field experiment data could be better choices for future investigations.

\section{Conclusions}

This paper develops an innovative high-order Markov chain model to predict particle dispersion and deposition under both constant ventilation mode and dynamic ventilation modes. The proposed models are validated against data from literature. Based on the results, the following conclusion can be drawn:

The proposed second-order Markov chain model has the capacity of, from a new perspective of probability-based state transfer, predicting the particle dispersion and particularly deposition during a limited number of consecutively changed ventilation modes with more reasonable accuracy but without significant computing cost increment compared with the first-order Markov chain model. In terms of reducing errors in particle deposition and escape prediction, the most suitable weight factors for simulation cases in the present study are found to be $\left(\lambda_{1}=0.7, \lambda_{2}=0.3, \lambda_{3}=0\right)$ for the second-order Markov chain model, and $\left(\lambda_{1}=0.8, \lambda_{2}=0.1\right.$, $\left.\lambda_{3}=0.1\right)$ for third-order Markov chain model. With the improvement of the efficiency of state transfer matrix construction as well as flow field related information acquisition, it is also believed that the proposed high-order
Markov chain model has the potential to perform fast (even real time) particle transport simulation under transient flow fields.

\section{Acknowledgements}

The investigation was supported by the National Science \& Technology Supporting Program (No. 2015BAJ03B00), the Natural Science Foundation of Hunan Province (Youth Program) (No. 2021JJ40591), the Doctoral Scientific Research Foundation of Changsha University of Science and Technology (No. 097/000301518), and the Scientific Research Project of Hunan Provincial Department of Education (No. 20C0033).

\section{References}

Abt E, Suh HH, Catalano P, et al. (2000). Relative contribution of outdoor and indoor particle sources to indoor concentrations. Environmental Science \& Technology, 34: 3579-3587.

Bouilly J, Limam K, Beghein C, et al. (2005). Effect of ventilation strategies on particle decay rates indoors: An experimental and modelling study. Atmospheric Environment, 39: 4885-4892.

Buonanno G, Marks GB, Morawska L (2013). Health effects of daily airborne particle dose in children: Direct association between personal dose and respiratory health effects. Environmental Pollution, 180: 246-250.

Chen C, Lin CH, Long Z, et al. (2014). Predicting transient particle transport in enclosed environments with the combined computational fluid dynamics and Markov chain method. Indoor Air, 24: 81-92.

Chen C, Liu W, Lin CH, et al. (2015). A Markov chain model for predicting transient particle transport in enclosed environments. Building and Environment, 90: 30-36.

Ching WK, Fung ES, Ng MK (2003). An higher-order Markov chain model for prediction of categorical data sequences. In: Ching WK, Ng MK (eds), Advances in Data Mining and Modeling, Singapore: World Scientific. pp. 129-141.

Chvatal V (1983). Linear Programming. New York: W. H. Freeman and Company.

Dai H, Zhao B (2020). Association of the infection probability of COVID-19 with ventilation rates in confined spaces. Building Simulation, 13: 1321-1327.

Deng Q, Ou C, Chen J, et al. (2018). Particle deposition in tracheobronchial airways of an infant, child and adult. Science of the Total Environment, 612: 339-346.

Díaz-Robles LA, Fu JS, Vergara-Fernández A, et al. (2014). Health risks caused by short term exposure to ultrafine particles generated by residential wood combustion: A case study of Temuco, Chile. Environment International, 66: 174-181.

Gao NP, Niu JL (2007). Modeling particle dispersion and deposition in indoor environments. Atmospheric Environment, 41: 3862-3876. 
Guelcher SA, Solomentsev Y, Anderson JL (2000). Aggregation of pairs of particles on electrodes during electrophoretic deposition. Powder Technology, 110: 90-97.

Holmberg S, Chen Q (2003). Air flow and particle control with different ventilation systems in a classroom. Indoor Air, 13: 200-204.

Howard-Reed C, Wallace LA, Emmerich SJ (2003). Effect of ventilation systems and air filters on decay rates of particles produced by indoor sources in an occupied townhouse. Atmospheric Environment, 37: 5295-5306.

Kan H, London SJ, Chen G, et al. (2007). Differentiating the effects of fine and coarse particles on daily mortality in Shanghai, China. Environment International, 33: 376-384.

Kim H, Kang K, Kim T (2020). CFD simulation analysis on make-up air supply by distance from cookstove for cooking-generated particle. International Journal of Environmental Research and Public Health, 17(21): 7799.

Klepeis NE, Nelson WC, Ott WR, et al. (2001). The National Human Activity Pattern Survey (NHAPS): A resource for assessing exposure to environmental pollutants. Journal of Exposure Science \& Environmental Epidemiology, 11: 231-252.

Lai ACK (2002). Particle deposition indoors: A review. Indoor Air, 12: 211-214.

Li F, Liu X, Liu J, et al. (2020). Solutions to mitigate the impact of measurement noise on the air pollution source strength estimation in a multi-zone building. Building Simulation, 13: 1329-1337.

Li Y, Chen Z (2003). A balance-point method for assessing the effect of natural ventilation on indoor particle concentrations. Atmospheric Environment, 37: 4277-4285.

Lin R-H, Wang C-R, Li C-S (1994). Size distributions and lung deposition of submicrometer particles from metered dose inhalers. Environment International, 20: 161-167.

Liu W, You R, Chen C (2019). Modeling transient particle transport by fast fluid dynamics with the Markov chain method. Building Simulation, 12: 881-889.

Lu H, Zhang L (2019). Effects of blockage ratio and Reynolds number on particle deposition in duct airflow over a forward-facing step. Building Simulation, 12: 1119-1129.

Lu W, Howarth AT, Adam N, et al. (1996). Modelling and measurement of airflow and aerosol particle distribution in a ventilated two-zone chamber. Building and Environment, 31: 417-423.

Mei X, Zhang T, Wang S (2016). Experimental investigation of jet-induced resuspension of indoor deposited particles. Aerosol Science and Technology, 50: 230-241.

Mei X, Gong G, Su H, et al. (2017). A grid-merging operation to accelerate the Markov chain model in predicting steady-state and transient transmission of airborne particles. Building and Environment, 122: 82-93.

Mei X, Gong G (2018). Predicting airborne particle deposition by a modified Markov chain model for fast estimation of potential contaminant spread. Atmospheric Environment, 185: 137-146.
Mei X, Gong G, Peng P, et al. (2019). Predicting thermophoresis induced particle deposition by using a modified Markov chain model. International Journal of Thermal Sciences, 136: 44-51.

Nazaroff WW (2004). Indoor particle dynamics. Indoor Air, 14(s7): $175-183$.

Nicas M (2000). Markov modeling of contaminant concentrations in indoor air. AIHAJ - American Industrial Hygiene Association, 61: 484-491.

Nomura Y, Hopke PK, Fitzgerald B, et al. (1997). Deposition of particles in a chamber as a function of ventilation rate. Aerosol Science and Technology, 27: 62-72.

Oravisjärvi K, Pietikäinen M, Ruuskanen J, et al. (2011). Effects of physical activity on the deposition of traffic-related particles into the human lungs in silico. Science of the Total Environment, 409: 4511-4518.

Rackes A, Waring MS (2013). Modeling impacts of dynamic ventilation strategies on indoor air quality of offices in six US cities. Building and Environment, 60: 243-253.

Rackes A, Waring MS (2014). Using multiobjective optimizations to discover dynamic building ventilation strategies that can improve indoor air quality and reduce energy use. Energy and Buildings, 75: 272-280.

Ray SK, Mohalik NK, Khan AM, et al. (2020). CFD modeling to study the effect of particle size on dispersion in 201 explosion chamber: an overview. International Journal of Mining Science and Technology, 30: 321-327.

Rim D, Novoselac A (2010). Ventilation effectiveness as an indicator of occupant exposure to particles from indoor sources. Building and Environment, 45: 1214-1224.

Rim D, Green M, Wallace L, et al. (2012). Evolution of ultrafine particle size distributions following indoor episodic releases: relative importance of coagulation, deposition and ventilation. Aerosol Science and Technology, 46: 494-503.

Satheesan MK, Mui KW, Wong LT (2020). A numerical study of ventilation strategies for infection risk mitigation in general inpatient wards. Building Simulation, 13: 887-896.

Shimada M, Okuyama K, Okazaki S, et al. (1996). Numerical simulation and experiment on the transport of fine particles in a ventilated room. Aerosol Science and Technology, 25: 242-255.

Sonnenberg AH, Herrmann J, Grinstaff MW, et al. (2020). A Markov chain model of particle deposition in the lung. Scientific Reports, 10: 13573.

Tsai C-J, Lu H-C (1995). Design and evaluation of a plate-to-plate thermophoretic precipitator. Aerosol Science and Technology, 22: $172-180$.

Wang M, Lin C-H, Chen Q (2012). Advanced turbulence models for predicting particle transport in enclosed environments. Building and Environment, 47: 40-49.

Wang Y, Li H, Feng G (2020). Simulating the influence of exhaust hood position on ultrafine particles during a cooking process in the residential kitchen. Building Simulation, 13: 1339-1352. 
Yang B, Ding X, Wang F, et al. (2021). A review of intensified conditioning of personal micro-environments: Moving closer to the human body. Energy and Built Environment, 2: 260-270.

Zhang Z, Chen Q (2004). Numerical analysis of particle behaviors in indoor air using lagrangian method. In: Proceedings of the 9th International Conference on Air Distribution in Rooms (Roomvent 2004), Coimbra, Portugal.

Zhang Z, Chen Q (2006). Experimental measurements and numerical simulations of particle transport and distribution in ventilated rooms. Atmospheric Environment, 40: 3396-3408.

Zhang R, Liu S, Zheng S (2021). Characterization of nano-to-micron sized respirable coal dust: Particle surface alteration and the health impact. Journal of Hazardous Materials, 413: 125447.

Zhao B, Zhang Y, Li X, et al. (2004). Comparison of indoor aerosol particle concentration and deposition in different ventilated rooms by numerical method. Building and Environment, 39: 1-8.

Zhao D, You X (2021). Cooking grease particles purification review and technology combination strategy evaluation for commercial kitchens. Building Simulation, 14: 1597-1617.

Zhou K, Li F, Cai H, et al. (2021). Experimental and numerical investigation of gas diffusion under an urban underground construction. Energy and Built Environment, 2: 436-444. 